\title{
RULED SURFACES WHOSE FLECNODE CURVES HAVE PLANE
}

\section{BRANCHES*}

\author{
BY
}

\section{A. F. CARPENTER}

\section{INTRODUCTION}

According to the point of view of Cayley, the curve of intersection of a surface with its tangent plane has a double point at the point of tangency. If this double point is also a point of inflection on one of the branches of the curve of intersection, Cayley very aptly calls such a surface point a flecnode $\dagger$ and speaks of the locus of all such points of a surface as its flecnode curve. Since the inflectional tangent at such a point must be regarded as having four coincident points in common with the curve, the flecnode curve may also be thought of as the locus of those points of a surface at which four-point tangents can be drawn. This point of view was first adopted by Salmon. $\ddagger$ It is the latter property of the flecnode curve with which we are here concerned.

On each generator of a ruled surface there will be in general two flecnodes since four consecutive generators of the surface have two straight line intersectors. In this sense the flecnode curve will be spoken of as having two branches. In the same way the flecnode surface, or locus of the four-point, or flecnode, tangents, will be a ruled surface of two sheets, one sheet through each branch of the flecnode curve.

In the general theory of ruled surfaces as developed by Wilczynski§ many important theorems are obtained by taking for directrix curves the two branches of the flecnode curve. It is the purpose of this paper to consider those ruled surfaces whose flecnode curves consist of two plane branches. Three cases will be considered: 1 , when the two branches are non-rectilinear and distinct; 2, when the two branches are non-rectilinear but coincident; 3 , when one, or both, branches are straight lines. As far as possible the

* Presented to the Society, December, 1914.

† Cayley, Collected Mathematical Papers, vol. II, p. 29.

¥Cambridge and Dublin Mathematical Journal, vol. IV, 1849, pp. 252-260.

$\S$ Wilczynski, Projective Differential Geometry of Curves and Ruled Surfaces. Hereafter referred to as W. 
notation used will be that already made familiar to mathematicians by the work of Professor Wilczynski to whose helpful suggestions and sympathy is due the inspiration for the work here presented.

The theory of ruled* surfaces may be based on the consideration of the system of ordinary linear homogeneous differential equations

$$
\begin{aligned}
& y^{\prime \prime}+p_{11} y^{\prime}+p_{12} z^{\prime}+q_{11} y+q_{12} z=0, \\
& z^{\prime \prime}+p_{21} y^{\prime}+p_{22} z^{\prime}+q_{21} y+q_{22} z=0,
\end{aligned}
$$

where $p_{i k}, q_{i k}$ are functions of $x$.

If $\left(y_{i}, z_{i}\right),(i=1, \cdots, 4)$ is a fundamental system of simultaneous solutions of $(A), y_{1}, \cdots, y_{4}$ and $z_{1}, \cdots, z_{4}$ are interpreted as the homogeneous coördinates of two points $P_{y}$ and $P_{z}$ of space. As $x$ varies $P_{y}$ and $P_{z}$ trace curves $C_{y}$ and $C_{z}$ between which there is evidently a point to point correspondence and which are directrix curves of the ruled surface $S$ generated by the line $L_{y z}$.

Every transformation of the form

$$
\eta=\alpha y+\beta z, \quad \zeta=\gamma y+\delta z, \quad \xi=f(x),
$$

where $\alpha, \beta, \gamma, \delta, f$ are arbitrary functions of $x$ and for which

$$
D=\alpha \delta-\beta_{\gamma} \neq 0,
$$

transforms $(A)$ into a new system of the same kind. Geometrically, (1) serves merely to replace the curves $C_{y}$ and $C_{z}$ by two other curves $C_{\eta}$ and $C_{\zeta}$ of $S$ and to change the parametric representation of the surface.

The differential equations of $C_{y}$ and $C_{z}$, that is, the differential equations satisfied respectively by $y_{1}, \cdots, y_{4}$ and $z_{1}, \cdots, z_{4}$, are in general of the fourth order. If either, say the one for $C_{y}$, is of the third order, then between $y_{1}, \cdots, y_{4}$ there exists one linear relation, that is, $C_{y}$ is a plane curve.

The necessary and sufficient conditions that $C_{y}$ and $C_{z}$ be plane curves, that is that their differential equations be of the third order, are $\nmid$ respectively

$$
\Delta_{1}=p_{12} s_{12}-q_{12} r_{12}=0, \quad \Delta_{2}=p_{21} s_{21}-q_{21} r_{21}=0
$$

where

$$
\begin{array}{ll}
r_{12}=p_{12}\left(p_{11}+p_{22}\right)-p_{12}^{\prime}-q_{12}, & r_{21}=p_{21}\left(p_{11}+p_{22}\right)-p_{21}^{\prime}-q_{21}, \\
s_{12}=p_{11} q_{12}+p_{12} q_{22}-q_{12}^{\prime}, & s_{21}=p_{21} q_{11}+p_{22} q_{21}-q_{21}^{\prime} .
\end{array}
$$

The fundamental invariants of system $(A)$ are $\ddagger$

$$
\Theta_{4}=I^{2}-4 J, \quad \Theta_{4.1}=8 \Theta_{4} \Theta_{4}^{\prime \prime}-9 \Theta_{4}^{\prime 2}+8 I \Theta_{4}^{2}, \quad \Theta_{10}=\left(I^{2}-4 J\right)\left(K-I^{\prime 2}\right)+\left(I I^{\prime}-2 J^{\prime}\right)^{2} \text {, }
$$

\footnotetext{
*W., p. 126 et seq.

$\dagger$ W., pp. 229, 230.

$\ddagger$ W., Ch. IV.
} 


$$
\Theta_{9}=\left|\begin{array}{rrr}
u_{11}-u_{22} & u_{12} & u_{21} \\
v_{11}-v_{22} & v_{12} & v_{21} \\
w_{11}-w_{22} & w_{12} & w_{21}
\end{array}\right|,
$$

where the quantities $u_{i k}$ are defined by the equations

$$
\begin{aligned}
& u_{11}=2 p_{11}^{\prime}-4 q_{11}+p_{11}^{2}+p_{12} p_{21}, \\
& u_{12}=2 p_{12}^{\prime}-4 q_{12}+p_{12}\left(p_{11}+p_{22}\right), \\
& u_{21}=2 p_{21}^{\prime}-4 q_{21}+p_{21}\left(p_{11}+p_{22}\right), \\
& u_{22}=2 p_{22}^{\prime}-4 q_{22}+p_{22}^{2}+p_{12} p_{21},
\end{aligned}
$$

where $v_{11}, v_{12}, v_{21}, v_{22}$, denote the quantities

and where

$$
\begin{aligned}
& v_{11}=2 u_{11}^{\prime}+p_{12} u_{21}-p_{21} u_{12}, \\
& v_{12}=2 u_{12}^{\prime}+\left(p_{11}-p_{22}\right) u_{12}-p_{12}\left(u_{11}-u_{22}\right), \\
& v_{21}=2 u_{21}^{\prime}-\left(p_{11}-p_{22}\right) u_{21}+p_{21}\left(u_{11}-u_{22}\right), \\
& v_{22}=2 u_{22}^{\prime}-p_{12} u_{21}+p_{21} u_{12}, \\
& w_{11}=2 v_{11}^{\prime}+p_{12} v_{21}-p_{21} v_{12}, \\
& w_{12}=2 v_{12}^{\prime}+\left(p_{11}-p_{22}\right) v_{12}-p_{12}\left(v_{11}-v_{22}\right), \\
& w_{21}=2 v_{21}^{\prime}-\left(p_{11}-p_{22}\right) v_{21}+p_{21}\left(v_{11}-v_{22}\right), \\
& w_{22}=2 v_{22}^{\prime}-p_{12} v_{21}+p_{21} v_{12},
\end{aligned}
$$

(5d) $I=u_{11}+u_{22}, \quad J=u_{11} u_{22}-u_{12} u_{21}, \quad K=v_{11} v_{22}-v_{12} v_{21}$.

A suitable transformation* on the dependent variables reduces $(A)$ to a new system which may be written without danger of confusion

(F) $y^{\prime \prime}+p_{12} z^{\prime}+q_{11} y+\frac{1}{2} p_{12}^{\prime} z=0, \quad z^{\prime \prime}+p_{21} y^{\prime}+\frac{1}{2} p_{21}^{\prime} y+q_{22} z=0$, and for which

$$
p_{11}=p_{22}=u_{12}=u_{21}=0 .
$$

When this transformation has been made the fundamental invariants reduce $\dagger$ to

$$
\begin{aligned}
\Theta_{4} & =\left(u_{11}-u_{22}\right)^{2}=16\left(q_{22}-q_{11}\right)^{2}, \\
\Theta_{4 \cdot 1} & =8 \Theta_{4} \Theta_{4}^{\prime \prime}-9 \Theta_{4}^{\prime 2}+8\left(u_{11}+u_{22}\right) \Theta_{4}^{2}, \\
\Theta_{9} & =2 \Theta_{4} \sqrt{\Theta_{4}}\left(p_{21} p_{12}^{\prime}-p_{12} p_{21}^{\prime}\right), \quad \Theta_{10}=\Theta_{4}^{2} p_{12} p_{21} .
\end{aligned}
$$

*W., pp. 116, 117.

†W., pp. 119, 120. 
If neither $\theta_{4}$ nor $\theta_{10}$ is zero, the coefficients of $(F)$ may be expressed in terms of the invariants by means of the formulæ

$$
\begin{aligned}
& p_{11}=0, \quad p_{22}=0, \quad q_{12}=\frac{1}{2} p_{22}^{\prime}, \quad q_{21}=\frac{1}{2} p_{21}^{\prime}, \\
& p_{12}=\frac{\sqrt{\Theta_{10}}}{\theta_{4}} e^{\frac{1}{4} \int \sqrt{\Theta_{4}}} \frac{\Theta_{4}}{\Theta_{10}} d x, \quad p_{21}=\frac{\sqrt{\theta_{10}}}{\theta_{4}} e^{-\frac{1}{t} \int \sqrt{\Theta_{4}} \frac{\Theta_{9}}{\Theta_{10}} d x}, \\
& 65 q_{11}=\frac{1}{\Theta_{4}^{2}}\left(16 \Theta_{10}-\Theta_{4 \cdot 1}+8 \Theta_{4} \Theta_{4}^{\prime \prime}-9{\Theta_{4}^{\prime 2}}^{2}\right)-8 \sqrt{\Theta_{4}}, \\
& 64 q_{22}=\frac{1}{\Theta_{4}^{2}}\left(16 \Theta_{10}-\Theta_{4 \cdot 1}+8 \Theta_{4} \Theta_{4}^{\prime \prime}-9{\Theta_{4}^{\prime}}^{2}\right)+8 \sqrt{\Theta_{4}} .
\end{aligned}
$$

Geometrically the reduction of system $(A)$ to the form $(F)$ is equivalent to choosing the two branches of the flecnode curve as directrix curves $C_{y}$ and $C_{z}$. This reduction can always be made if $\theta_{4} \neq 0$, i. e., if the flecnode curve has distinct branches.* It is with system $(F)$ that we shall deal in part 1 .

I. The two bRanches of the Flecnode CURVE ARE Distinct, NONRECTILINEAR, PLANE CURVES

By means of $(8 a)$, equations (3) become

$$
\begin{array}{ll}
r_{12}=-{ }_{2}^{3} p_{12}^{\prime}, & s_{12}=p_{12} q_{22}-\frac{1}{2} p_{12}^{\prime \prime}, \\
r_{21}=-\frac{3}{2} p_{21}^{\prime}, & s_{21}=p_{21} q_{11}-\frac{1}{2} p_{21}^{\prime \prime},
\end{array}
$$

and by substituting from (8a) and (9) in (2) we obtain the necessary and sufficient conditions that $C_{y}$ and $C_{z}$ shall be plane curves, in the form

(10) $\Delta_{1}=p_{12}^{2} q_{22}-\frac{1}{2} p_{12} p_{12}^{\prime \prime}+\frac{3}{4} p_{12}^{\prime 2}=0, \quad \Delta_{2}=p_{21}^{2} q_{11}-\frac{1}{2} p_{21} p_{21}^{\prime \prime}+\frac{3}{4} p_{21}^{\prime 2}=0$.

We have from $(8 b)$ by differentiation

$$
\begin{aligned}
& p_{12}^{\prime}=\frac{p_{12}}{4 \Theta_{4} \Theta_{10}}\left(\Theta_{4} \sqrt{\Theta_{4}} \Theta_{9}+2 \Theta_{4} \Theta_{10}^{\prime}-4 \Theta_{4}^{\prime} \Theta_{10}\right) \\
& p_{21}^{\prime}=\frac{p_{21}}{4 \theta_{4} \theta_{10}}\left(-\theta_{4} \sqrt{\Theta_{4}} \theta_{9}+2 \theta_{4} \theta_{10}^{\prime}-4 \theta_{4}^{\prime} \theta_{10}\right), \\
& \text { (11) } p_{12}^{\prime \prime}=\frac{p_{12}}{16 \Theta_{4}^{2} \theta_{10}^{2}}\left(\theta_{4}^{3} \theta_{9}^{2}-4 \theta_{4}^{2}{\Theta_{10}^{\prime 2}}^{\prime 2} 32{\theta_{4}^{\prime 2}}^{2} \theta_{10}^{2}-6 \Theta_{4} \sqrt{\Theta_{4}} \theta_{4}^{\prime} \theta_{9} \Theta_{10}\right. \\
& \left.-16 \theta_{4} \theta_{4}^{\prime} \theta_{10} \theta_{10}^{\prime}+4 \theta_{4}^{2} \sqrt{\theta_{4}} \theta_{9}^{\prime} \theta_{10}+8 \theta_{4}^{2} \theta_{10} \theta_{10}^{\prime \prime}-16 \theta_{4} \theta_{4}^{\prime \prime} \theta_{10}^{2}\right), \\
& p_{21}^{\prime \prime}=\frac{p_{21}}{16 \theta_{4}^{2} \theta_{10}^{2}}\left(\Theta_{4}^{3} \Theta_{9}^{2}-4 \Theta_{4}^{2}{\Theta_{10}^{\prime 2}}^{\prime 2}+32{\theta_{4}^{\prime}}^{2} \Theta_{10}^{2}+6 \Theta_{4} \sqrt{\Theta_{4}} \Theta_{4}^{\prime} \theta_{9} \theta_{10}\right. \\
& \left.-16 \theta_{4} \theta_{4}^{\prime} \theta_{10} \theta_{10}^{\prime}-4 \theta_{4}^{2} \sqrt{\Theta_{4}} \theta_{9}^{\prime} \theta_{10}+8 \theta_{4}^{2} \theta_{10} \theta_{10}^{\prime \prime}-16 \theta_{4} \theta_{4}^{\prime \prime} \theta_{10}^{2}\right) \text {. }
\end{aligned}
$$

*W., pp. 148, 149. The necessary and sufficient condition that $C_{y}$ and $C_{z}$ be the two branches of the flecnode curve is $u_{12}=u_{21}=0$. 
By substituting from (8b) and (11) in (10) we obtain $\Delta_{1}$ and $\Delta_{2}$ in terms of the fundamental invariants, in the form

where

$$
\Delta_{1}=\frac{p_{12}^{2} \delta_{1}}{64 \theta_{4}^{2} \Theta_{10}^{2}}=0, \quad \Delta_{2}=\frac{p_{21}^{2} \delta_{2}}{64 \Theta_{4}^{2} \Theta_{10}^{2}}=0
$$

$$
\begin{gathered}
\delta_{1}=16 \theta_{10}^{3}-\theta_{4 \cdot 1} \theta_{10}^{2}+40 \theta_{4} \theta_{4}^{\prime \prime} \theta_{10}^{2}-25 \theta_{4}^{\prime 2} \theta_{10}^{2}+\theta_{4}^{3} \theta_{9}^{2}+20 \theta_{4}^{2} \theta_{10}^{\prime 2} \\
-16 \theta_{4} \theta_{4}^{\prime} \theta_{10} \theta_{10}^{\prime}-16 \theta_{4}^{2} \theta_{10} \theta_{10}^{\prime \prime}+4 \theta_{4} \sqrt{\theta_{4}}\left(2 \theta_{4} \theta_{10}^{2}-3 \theta_{4}^{\prime} \theta_{9} \theta_{10}\right. \\
\left.-2 \theta_{4} \theta_{9}^{\prime} \theta_{10}+3 \theta_{4} \theta_{9} \theta_{10}^{\prime}\right), \\
\delta_{2}=16 \theta_{10}^{3}-\theta_{4 \cdot 1} \theta_{10}^{2}+40 \theta_{4} \theta_{4}^{\prime \prime} \theta_{10}^{2}-25 \theta_{4}^{\prime 2} \theta_{10}^{2}+\theta_{4}^{3} \theta_{9}^{2} \\
+20 \theta_{4}^{2} \theta_{10}^{\prime 2}-16 \theta_{4} \theta_{4}^{\prime} \theta_{10} \theta_{10}^{\prime}-16 \theta_{4}^{2} \theta_{10} \theta_{10}^{\prime \prime}-4 \theta_{4} \sqrt{\theta_{4}}\left(2 \theta_{4} \theta_{10}^{2}\right. \\
\left.-3 \theta_{4}^{\prime} \theta_{9} \theta_{10}-2 \theta_{4} \theta_{9}^{\prime} \theta_{10}+3 \theta_{4} \theta_{9} \theta_{10}^{\prime}\right) .
\end{gathered}
$$

Now $p_{12}$ can not be zero for otherwise the first of equations $(F)$ would reduce to a second order equation in $y$ alone, that is, $C_{y}$ would be a straight line contrary to our hypothesis. Similarly for $p_{21}$. We conclude therefore that $\delta_{1}=0$ and $\delta_{2}=0$ are the conditions for a flecnode curve with two distinct nonrectilinear plane branches.

It should be noted that $\delta_{1}$ and $\delta_{2}$ are invariants of weight thirty. We have*

$$
\begin{array}{ll}
\theta_{14}=9 \theta_{9} \theta_{4}^{\prime}-4 \theta_{4} \theta_{9}^{\prime}, & \theta_{15}=5 \theta_{10} \theta_{4}^{\prime}-2 \theta_{4} \theta_{10}^{\prime}, \\
\theta_{20}=15 \theta_{15} \theta_{4}^{\prime}-4 \theta_{4} \theta_{15}^{\prime}, & \vartheta_{20}=10 \Theta_{10} \theta_{9}^{\prime}-9 \Theta_{9} \theta_{10}^{\prime} .
\end{array}
$$

From (12) we find

$$
\begin{aligned}
\delta_{1}-\delta_{2}= & 8 \theta_{4} \sqrt{\Theta_{4}}\left(3 \theta_{4} \theta_{9} \theta_{10}^{\prime}-2 \theta_{4} \theta_{9}^{\prime} \theta_{10}-3 \theta_{4}^{\prime} \theta_{9} \theta_{10}+2 \theta_{4} \theta_{10}^{2}\right), \\
\delta_{1}+\delta_{2}=2 & \left(16 \theta_{10}^{3}+\theta_{4}^{3} \theta_{9}^{2}-\theta_{4 \cdot 1} \theta_{10}^{2}+40 \theta_{4} \theta_{4}^{\prime \prime} \theta_{10}^{2}\right. \\
& \left.-16 \theta_{4}^{2} \theta_{10} \theta_{10}^{\prime \prime}+20 \theta_{4}^{2} \theta_{10}^{\prime 2}-16 \theta_{4} \theta_{4}^{\prime} \theta_{10} \theta_{10}^{\prime}-25 \theta_{4}^{\prime 2} \theta_{10}^{2}\right),
\end{aligned}
$$

or, by making use of (13),

$$
\begin{aligned}
& \delta_{1}-\delta_{2}=-\frac{8}{3} \theta_{4} \sqrt{\Theta_{4}}\left(\theta_{10} \theta_{14}+\theta_{4} \theta_{20}-6 \theta_{4} \theta_{10}^{2}\right), \\
& \delta_{1}+\delta_{2}=2\left(16 \theta_{10}^{3}+\theta_{4}^{3} \theta_{9}^{2}-\theta_{4 \cdot 1} \theta_{10}^{2}+5 \theta_{15}^{2}+2 \theta_{10} \vartheta_{20}\right),
\end{aligned}
$$

whence

$$
\begin{aligned}
\delta_{1}=16 \theta_{10}^{3}+\theta_{4}^{3} \theta_{9}^{2}-\theta_{4 \cdot 1} \theta_{10}^{2} & +5 \theta_{15}^{2}+2 \theta_{10} \vartheta_{20} \\
& -\frac{4}{3} \theta_{4} \sqrt{\theta_{4}}\left(\theta_{10} \theta_{14}+\theta_{4} \theta_{20}-6 \theta_{4} \theta_{10}^{2}\right), \\
\delta_{2}=16 \theta_{10}^{3}+\theta_{4}^{3} \theta_{9}^{2}-\theta_{4 \cdot 1} \theta_{10}^{2} & +5 \theta_{15}^{2}+2 \theta_{10} \vartheta_{20} \\
& +\frac{4}{3} \theta_{4} \sqrt{\theta_{4}}\left(\theta_{10} \theta_{14}+\theta_{4} \theta_{20}-6 \theta_{4} \theta_{10}^{2}\right),
\end{aligned}
$$

which equations put the invariance of $\delta_{1}$ and $\delta_{2}$ in evidence.

$$
\text { *W., p. } 112 \text {. }
$$


The differential equation for $C_{y}$ is, ${ }^{*}$ in view of $(8 a)$

$$
y^{\prime \prime \prime}+3 p_{1} y^{\prime \prime}+3 p_{2} y^{\prime}+p_{3} y=0,
$$

where

$$
\begin{gathered}
p_{1}=-\frac{p_{12}^{\prime}}{2 p_{12}}, \quad p_{2}=-\frac{1}{3}\left(p_{12} p_{21}-q_{11}\right), \\
p_{3}=-\frac{3 p_{12}^{\prime} q_{11}}{2 p_{12}}-\frac{1}{2} p_{12} p_{21}^{\prime}+q_{11}^{\prime} .
\end{gathered}
$$

The invariant of weight three for this curve may now be calculated. It is where $\dagger$

$$
\Theta_{3}=P_{3}-\frac{3}{2} P_{2}^{\prime},
$$

$$
P_{2}=p_{2}-p_{1}^{2}-p_{1}^{\prime}, \quad P_{3}=p_{3}-3 p_{1} p_{2}+2 p_{1}^{3}-p_{1}^{\prime \prime} .
$$

By making use of (17), and of the first of (10) to eliminate derivatives of higher order than the first, we find that

$$
\Theta_{3}=\frac{p_{12}^{\prime}}{p_{12}}\left(q_{22}-q_{11}\right)-\frac{1}{2}\left(q_{22}^{\prime}-q_{11}^{\prime}\right),
$$

or, in view of $(8 b)$ and (13)

$$
\theta_{3}=\frac{\theta_{4}^{2} \theta_{9}-\sqrt{\theta_{4}} \theta_{15}}{16 \theta_{4} \theta_{10}}
$$

In similar manner we obtain the corresponding invariant for $C_{z}$. It is

$$
\Theta_{3}=\frac{p_{21}^{\prime}}{p_{21}}\left(q_{11}-q_{22}\right)-\frac{1}{2}\left(q_{11}^{\prime}-q_{22}^{\prime}\right)=\frac{\Theta_{4}^{2} \Theta_{9}+\sqrt{\Theta_{4}} \Theta_{15}}{16 \Theta_{4} \Theta_{10}} .
$$

These invariants of $C_{y}$ and $C_{z}$ are also invariants of weight three of the surface $S$.

Let us suppose that $C_{y}$ is a conic, $\ddagger$ that is, that $\theta_{3}=0$. We have from (19)

If $C_{z}$ is a conic, we find

$$
\theta_{4}^{\frac{3}{2}} \theta_{9}-\theta_{15}=0 \text {. }
$$

$$
\theta_{4}^{\frac{3}{2}} \theta_{9}+\theta_{15}=0,
$$

so that if either $C_{y}$ or $C_{z}$ is a conic we shall have the rational invariant equation

$$
\theta_{4}^{3} \theta_{9}^{2}-\theta_{15}^{2}=0 \text {. }
$$

But Wilczynski has shown that any ruled surface which satisfies this condition has the property that the principal surface of the flecnode congruence intersects one of the sheets of the flecnode surface along the second branch of

*W., p. 230.

$\dagger W .$, p. 59.

$\ddagger W .$, p. 61. 
its flecnode curve.* We conclude therefore that if either branch of the flecnode curve of a ruled surface is a conic then the principal surface of the flecnode congruence intersects one of the sheets of the flecnode surface along the second branch of its flecnode curve. $\dagger$ Moreover this theorem holds when only one branch of the flecnode curve is plane.

Let us next suppose that both $C_{y}$ and $C_{z}$ are conics. By addition of (18) and (20) we have

$$
\left(\frac{p_{12}^{\prime}}{p_{12}}-\frac{p_{21}^{\prime}}{p_{21}}\right)\left(q_{22}-q_{11}\right)=0 .
$$

But if $q_{22}-q_{11}=0$, then by (7), $\theta_{4}=0$, that is the two branches of the flecnode curve are coincident, $\ddagger$ contrary to our hypothesis. It follows that $p_{12}^{\prime} / p_{12}=p_{21}^{\prime} / p_{21}$ and therefore that

$$
\begin{aligned}
\frac{d}{d x}\left(\frac{p_{12}^{\prime}}{p_{12}}\right)-\frac{1}{2}\left(\frac{p_{12}^{\prime}}{p_{12}}\right)^{2}=\frac{p_{12}^{\prime \prime}}{p_{12}}-\frac{3}{2}\left(\frac{p_{12}^{\prime}}{p_{12}}\right)^{2}= & \frac{d}{d x}\left(\frac{p_{21}^{\prime}}{p_{21}}\right) \\
& -\frac{1}{2}\left(\frac{p_{21}^{\prime}}{p_{21}}\right)^{2}=\frac{p_{21}^{\prime \prime}}{p_{21}}-\frac{3}{2}\left(\frac{p_{21}^{\prime}}{p_{21}}\right)^{2} .
\end{aligned}
$$

Now from (10) we have

$$
2 q_{22}=\frac{p_{12}^{\prime \prime}}{p_{12}}-\frac{3}{2}\left(\frac{p_{12}^{\prime}}{p_{12}}\right)^{2}, \quad 2 q_{11}=\frac{p_{21}^{\prime \prime}}{p_{21}}-\frac{3}{2}\left(\frac{p_{21}^{\prime}}{p_{21}}\right)^{2}
$$

and this, with (21) gives again $q_{22}-q_{11}=0$. We conclude therefore that there are no ruled surfaces whose flecnode curves are composed of two distinct, non-degenerate, conics.

It is possible, by a suitably chosen transformation on the independent variable, $\S$ to make $u_{11}-u_{22}=1$.

Let us assume that system $(F)$ is such that

$$
p_{11}=p_{22}=u_{12}=u_{21}=0, \quad u_{11}-u_{22}=1,
$$

and at the same time that $C_{y}$ is a conic while $C_{z}$ is any plane curve not a conic. From equations (7) we have

$$
\Theta_{4}=1,
$$

and from (19) and (13) it then follows, since $C_{y}$ is a conic, that

$$
\theta_{9}-\theta_{15}=\theta_{9}+2 \theta_{10}^{\prime}=0 .
$$

*W., p. 175 and pp. 216, 217.

$\dagger$ If $F^{\prime}$ and $F^{\prime \prime}$ are the two sheets of the flecnode surface of $S$ then $S$ is one sheet of the flecnode surface of $F^{\prime}\left(F^{\prime \prime}\right)$ and $C_{y}\left(C_{z}\right)$ is one branch of the flecnode curve of $F^{\prime}\left(F^{\prime \prime}\right)$.

$\ddagger W$., p. 150.

$\S$ W., p. 117 .

Trans. Am. Math. Soc. 34 
By means of (23) and (24) the first of equations (12) gives

$$
\begin{aligned}
\delta_{1}=\theta_{10}^{2}\left[8\left(2 \theta_{10}+1\right)-\right. & \left.\theta_{4 \cdot 1}\right]+\left(\theta_{9}+2 \theta_{10}^{\prime}\right)\left(\theta_{9}+10 \theta_{10}^{\prime}\right) \\
& -8 \theta_{10}\left(\theta_{9}^{\prime}+2 \theta_{10}^{\prime \prime}\right)=\theta_{10}^{2}\left[8\left(2 \theta_{10}+1\right)-\theta_{4 \cdot 1}\right]
\end{aligned}
$$

But in our case $\delta_{1}=0$ and $\theta_{10} \neq 0$, so that we find

$$
\theta_{4 \cdot 1}=8\left(2 \theta_{10}+1\right) \text {. }
$$

Remembering also that $\delta_{2}=0$, the first of equations (14) may be reduced by means of (23) and (24) to

$$
2 \theta_{10} \theta_{10}^{\prime \prime}-3 \Theta_{10}^{\prime 2}+\theta_{10}^{2}=0 .
$$

The general solution of $(26)$ is

$$
\Theta_{10}=\frac{4 A_{2} e^{x}}{A_{1}\left(A_{2}-e^{x}\right)^{2}},
$$

and this result substituted in (24) and (25) gives

$$
\Theta_{9}=-\frac{8 A_{2} e^{x}\left(A_{2}+e^{x}\right)}{A_{1}\left(A_{2}-e^{x}\right)^{3}}, \quad \Theta_{4 \cdot 1}=8\left[\frac{8 A_{2} e^{x}}{A_{1}\left(A_{2}-e^{x}\right)^{2}}+1\right] .
$$

We now have our four fundamental invariants expressed explicitly in terms of the independent variable and two arbitrary constants. Consequently it is possible to calculate the coefficients of system $(F)$. By means of (23) and the first of equations ( $8 b)$, we find $p_{12}=A_{3}$. The last equations ( 7 ) then gives

From $(8 a)$ we find

$$
p_{21}=\frac{\Theta_{10}}{p_{12}}=\frac{4 A_{2} e^{x}}{A_{1} A_{3}\left(A_{2}-e^{x}\right)^{2}} .
$$

$$
q_{12}=0, \quad q_{21}=\frac{2 A_{2} e^{x}\left(A_{2}+e^{x}\right)}{A_{1} A_{3}\left(A_{2}-e^{x}\right)^{3}} .
$$

From the first of equations (10), since $p_{12}$ is a constant, $q_{22}=0$, and since $u_{11}-u_{22}=4\left(q_{22}-q_{11}\right)=1$,

$$
q_{11}=-\frac{1}{4} .
$$

The coefficients of system $(F)$ have now been calculated. They involve three arbitrary constants of integration, but two of these are not essential; for the transformation

$$
\eta=\frac{y}{A_{3}}, \quad \zeta=z, \quad \xi=x-\log A_{2},
$$

leaves conditions (22) unchanged and serves to remove $A_{2}$ and $A_{3}$. The 
transformed system of equations is

$$
\eta^{\prime \prime}+\zeta^{\prime}-\frac{1}{4} \eta=0, \quad \zeta^{\prime \prime}+\frac{2 A e^{\xi}}{\left(1-e^{\xi}\right)^{2}} \eta^{\prime}+\frac{A e^{\xi}\left(1+e^{\xi}\right)}{\left(1-e^{\xi}\right)^{3}} \eta=0
$$

Since each value of $A$ gives a system of equations defining a class of mutually projective ruled surfaces, ${ }^{*}$ we conclude that there exists a single infinity of classes of mutually projective ruled surfaces having the property that their flecnode curves consist of two distinct plane branches, one of which is a conic.

Closely connected with the flecnode curve of a ruled surface is its complex curve defined by means of the linear complexes which osculate $S$ along its generators. Wilczynski has shown $\dagger$ that in general the complex curve cuts each generator of $S$ in two points and that these two points are harmonically separated by the two flecnode points. The transformation which serves to replace the pair of directrix curves associated with system $(A)$, with the two branches of the complex curve, is determined by the two factors of the covariant $\ddagger$

$$
\begin{aligned}
& {\left[\left(u_{11}-u_{22}\right) v_{12}-\left(v_{11}-v_{22}\right) u_{12}\right] z^{2}+\left[\left(u_{11}-u_{22}\right) v_{21}\right.} \\
& \left.-\left(v_{11}-v_{22}\right) u_{21}\right] y^{2}+2\left(u_{12} v_{21}-u_{21} v_{12}\right) y z .
\end{aligned}
$$

For system $(F)$ this covariant reduces to

$$
\left(u_{11}-u_{22}\right)^{2}\left(p_{21} y^{2}-p_{12} z^{2}\right),
$$

so that the transformation which replaces the two branches of the flecnode curve with the two branches of the complex curve is

$$
\bar{y}=\sqrt{p_{21}} y+\sqrt{p_{12}} z, \quad \bar{z}=\sqrt{p_{21}} y-\sqrt{p_{12}} z .
$$

If we denote by $\bar{p}_{i k}, \bar{q}_{i k},(i, k=1,2)$, the coefficients of this new system satisfied by $\bar{y}$ and $\bar{z}$, we have

$$
\begin{gathered}
\bar{p}_{11}=\sqrt{p_{12} p_{21}}-\frac{1}{2}\left(\frac{p_{12}^{\prime}}{p_{12}}+\frac{p_{21}^{\prime}}{p_{21}}\right), \quad \bar{p}_{22}=-\sqrt{p_{12} p_{21}}-\frac{1}{2}\left(\frac{p_{12}^{\prime}}{p_{12}}+\frac{p_{21}^{\prime}}{p_{21}}\right), \\
\bar{p}_{12}=\bar{p}_{21}=\frac{1}{2}\left(\frac{p_{12}^{\prime}}{p_{12}}-\frac{p_{21}^{\prime}}{p_{21}}\right), \\
\bar{q}_{11}=\bar{q}_{22}=\frac{1}{2}\left(\frac{\Delta_{2}}{p_{21}^{2}}+\frac{\Delta_{1}}{p_{12}^{2}}\right), \quad \bar{q}_{12}=\bar{q}_{21}=\frac{1}{2}\left(\frac{\Delta_{2}}{p_{21}^{2}}-\frac{\Delta_{1}}{p_{12}^{2}}\right) .
\end{gathered}
$$

If, as we suppose, $C_{y}$ and $C_{z}$ are plane curves, then according to (10)

$$
\bar{q}_{11}=\bar{q}_{22}=\bar{q}_{12}=\bar{q}_{21}=0 \text {, }
$$

*W., p. 132.

†W., pp. 204-206.

$\ddagger W .$, p. 208. 
and we may write our new system of equations in the form

$$
\begin{aligned}
& \bar{y}^{\prime \prime}+\left[\sqrt{p_{12} p_{21}}-\frac{1}{2}\left(\frac{p_{12}^{\prime}}{p_{12}}+\frac{p_{: 21}^{\prime}}{p_{21}}\right)\right] \bar{y}^{\prime}+\frac{1}{2}\left(\frac{p_{12}^{\prime}}{p_{12}}-\frac{p_{21}^{\prime}}{p_{21}}\right) \bar{z}^{\prime}=0, \\
& \bar{z}^{\prime \prime}+{ }_{2}^{1}\left(\frac{p_{12}^{\prime}}{p_{12}}-\frac{p_{21}^{\prime}}{p_{21}}\right) \bar{y}^{\prime}-\left[\sqrt{p_{12} p_{21}}+\frac{1}{2}\left(\frac{p_{12}^{\prime}}{p_{12}}+\frac{p_{21}^{\prime}}{p_{21}}\right)\right] \bar{z}^{\prime}=0 .
\end{aligned}
$$

The differential equations for $C_{v}$ and $C_{z}$ may now be found. If we include with the two equations $(C)$ the equation obtained by differentiating the first of the pair, we may eliminate $\bar{z}^{\prime \prime}$ and $\bar{z}^{\prime}$ from this set of three equations and obtain

$$
\begin{aligned}
& \bar{y}^{\prime \prime \prime}+\frac{1}{\bar{p}_{12}}\left[\left(\bar{p}_{11}+\bar{p}_{22}\right) \bar{p}_{12}-\bar{p}_{12}^{\prime}\right] \bar{y}^{\prime \prime} \\
& +\frac{1}{\bar{p}_{12}}\left(\bar{p}_{11} \bar{p}_{12} \bar{p}_{22}-\bar{p}_{11} \bar{p}_{12}^{\prime}+\bar{p}_{11}^{\prime} \bar{p}_{12}-\bar{p}_{12}^{3}\right) \bar{y}^{\prime}=0 .
\end{aligned}
$$

By a similar process

$$
\begin{aligned}
& \bar{z}^{\prime \prime \prime}+\frac{1}{\bar{p}_{12}}\left[\left(\bar{p}_{11}+\bar{p}_{22}\right) \bar{p}_{12}-\bar{p}_{12}^{\prime}\right] \bar{z}^{\prime \prime} \\
& +\frac{1}{\bar{p}_{12}}\left(p_{11} \bar{p}_{12} \bar{p}_{22}-\bar{p}_{12}^{\prime} \bar{p}_{22}+\bar{p}_{12} \bar{p}_{22}^{\prime}-\bar{p}_{12}^{3}\right) \bar{z}^{\prime}=0 . *
\end{aligned}
$$

Since (31) and (32) are of the third order only, we conclude that if the two branches of the flecnode curve are distinct, non-rectilinear, plane curves, then the two branches of the complex curve are distinct, non-rectilinear, plane curves.

We now wish to ascertain the relation which the planes of the two branches of the complex curve bear to the planes of the two branches of the flecnode curve. For this purpose we choose, with Wilczynski, the tetrahedron $P_{y} P_{z} P_{\rho} P_{\sigma}$ for tetrahedron of reference, where

$$
\rho=2 y^{\prime}+p_{11} y+p_{12} z, \quad \sigma=2 z^{\prime}+p_{21} y+p_{22} z,
$$

are semi-covariants of system $(A)$ which reduce to

$$
\rho=2 y^{\prime}+p_{12} z, \quad \sigma=2 z^{\prime}+p_{21} y,
$$

for system $(F)$. Moreover we may choose the local coördinate system associated with $P_{y} P_{z} P_{\rho} P_{\sigma}$ in such a way that the point represented by the expression $\lambda y+\mu z+\nu \rho+\chi \sigma$ will have the coördinates $(\lambda, \mu, \nu, \chi) . \dagger$

Referred to the tretrahedron $P_{y} P_{z} P_{\rho} P_{\sigma}$ the equation of the plane oscu-

${ }^{*} \bar{p}_{12} \neq 0$ for otherwise we would have $p_{12}=k p_{21}$ and it would follow from (10) that $q_{11}-q_{22}=0$, i.e., $\Theta_{4}=0$.

$\dagger$ This amounts to choosing the point represented by $y+z+\rho+\sigma$ as unit point. 
lating $C_{\bar{y}}$ at $P_{y}$ is

$$
\left|\begin{array}{llll}
x_{1} & x_{2} & x_{3} & x_{4} \\
\bar{y}_{1} & \bar{y}_{2} & \bar{y}_{3} & \bar{y}_{4} \\
\bar{y}_{1}^{\prime} & \bar{y}_{2}^{\prime} & \bar{y}_{3}^{\prime} & \bar{y}_{4}^{\prime} \\
\bar{y}_{1}^{\prime \prime} & \bar{y}_{2}^{\prime \prime} & \bar{y}_{3}^{\prime \prime} & \bar{y}_{4}^{\prime \prime}
\end{array}\right|=0 .
$$

But this is also the equation of the plane of $C_{\bar{y}}$. We have

$$
\bar{y}_{k}=\sqrt{p_{21}} y_{k}+\sqrt{p_{12}} z_{k} .
$$

Making use of (34) and (10)

$$
\begin{aligned}
& 2 \bar{y}_{k}^{\prime}=\left(\frac{p_{21}^{\prime}}{\sqrt{p_{21}}}-\sqrt{p_{12}} p_{21}\right) y_{k}+\left(\frac{p_{12}^{\prime}}{\sqrt{p_{12}}}-p_{12} \sqrt{p_{21}}\right) z_{k}+\sqrt{p_{21}} \rho_{k}+\sqrt{p_{12}} \sigma_{k}, \\
& 2 \bar{y}_{k}^{\prime \prime}=\left(p_{12} p_{21} \sqrt{p_{21}}-\sqrt{p_{12}} p_{21}^{\prime}-\frac{p_{21} p_{12}^{\prime}}{\sqrt{p_{12}}}+\frac{p_{21}^{\prime 2}}{p_{21} \sqrt{p_{21}}}\right) y_{k} \\
& +\left(p_{12} \sqrt{p_{12}} p_{21}-p_{12}^{\prime} \sqrt{p_{21}}-\frac{p_{12} p_{21}^{\prime}}{\sqrt{p_{21}}}+\frac{p_{12}^{\prime 2}}{p_{12} \sqrt{p_{12}}}\right) z_{k} \\
& +\left(\frac{p_{21}^{\prime}}{\sqrt{p_{21}}}-\sqrt{p_{12}} p_{21}\right) \rho_{k}+\left(\frac{p_{12}^{\prime}}{\sqrt{p_{12}}}-p_{12} \sqrt{p_{21}}\right) \sigma_{k} .
\end{aligned}
$$

By means of these values, (35) becomes, after dividing by $p_{21} p_{12}^{\prime}-p_{12} p_{21}^{\prime}$,*

$$
\frac{1}{\sqrt{p_{21}}} x_{1}-\frac{1}{\sqrt{p_{12}}} x_{2}-\left(\sqrt{p_{12}}+\frac{p_{21}^{\prime}}{p_{21} \sqrt{p_{21}}}\right) x_{3}+\left(\sqrt{p_{21}}+\frac{p_{12}^{\prime}}{p_{12} \sqrt{p_{12}}}\right) x_{4}=0
$$

In similar mannert we obtain the equation of the plane of $C_{z}$ in the form

$$
\frac{1}{\sqrt{p_{21}}} x_{1}+\frac{1}{\sqrt{p_{12}}} x_{2}+\left(\sqrt{p_{12}}-\frac{p_{21}^{\prime}}{p_{21} \sqrt{p_{21}}}\right) x_{3}+\left(\sqrt{p_{21}}-\frac{p_{12}^{\prime}}{p_{12} \sqrt{p_{12}}}\right) x_{4}=0 .
$$

Now the equations of the planes of $C_{y}$ and $C_{z}$ aref respectively

$$
\begin{aligned}
& p_{12} x_{2}+p_{12}^{2} x_{3}-p_{12}^{\prime} x_{4}=0, \\
& p_{21} x_{1}-p_{21}^{\prime} x_{3}+p_{21}^{2} x_{4}=0 .
\end{aligned}
$$

If (36) be subtracted from (37) and the resulting equation multiplied by $\frac{1}{2} p_{12} \sqrt{p_{12}},(38)$ is obtained, while if $(36)$ and (37) are added and the resulting equations multiplied by $\frac{1}{2} p_{21} \sqrt{p_{21}},(39)$ is obtained. We conclude therefore that the planes of the two branches of the complex curve pass through the line of

${ }^{*} p_{21} p_{12}^{\prime}-p_{12} p_{21}^{\prime} \neq 0$. See footnote p. 518 .

$\dagger$ In view of (29), (37) may be obtained from (36) by changing the sign of $\sqrt{p_{12}}$.

$\ddagger$ W., p. 214. (38) and (39) are more generally the planes osculating $C_{y}$ and $C_{z}$ at $P_{y}$ and $P_{z}$. 
intersection of the planes of the two branches of the flecnode curve. Combining the last two results with the harmonic property of the points $P_{y}, P_{\bar{y}}, P_{z}, P_{\bar{z}}$, we are able to state the following

THeOREM I. If the two branches of the flecnode curve of a ruled surface are distinct, non-rectilinear, plane curves, then the two branches of the complex curve have this same property and the four planes of these curves form a harmonic pencil of planes in which the planes of the two branches of the flecnode curve are separated by the planes of the two branches of the complex curve.

We proceed next to investigate the converse of Theorem I. It is evident from the start that if either $p_{12}=0$ or $p_{21}=0, C_{\bar{y}}$ and $C_{\bar{z}}$ will coincide with one or other branch of the flecnode curve by (29) and that this branch will be a straight line by $(8 a)$ and $(F)$. The necessary and sufficient conditions that $C_{\bar{y}}$ and $C_{\Sigma}$ shall be distinct plane curves are, by (2) and (30)

(40) $\bar{\Delta}_{1}=\bar{p}_{12} \bar{s}_{12}-\bar{q}_{12} \bar{r}_{12}=\bar{p}_{12}^{2} \bar{q}_{11}-\bar{p}_{12} \bar{p}_{22} \bar{q}_{12}+\bar{q}_{12}^{2}+\bar{p}_{12}^{\prime} \bar{q}_{12}-\bar{p}_{12} \bar{q}_{12}^{\prime}=0$,

(41) $\bar{\Delta}_{2}=\bar{p}_{21} \bar{s}_{21}-\bar{q}_{21} \bar{r}_{21}=\bar{p}_{12}^{2} \bar{q}_{11}-\bar{p}_{11} \bar{p}_{12} \bar{q}_{12}+\bar{q}_{12}^{2}+\bar{p}_{12}^{\prime} \bar{q}_{12}-\bar{p}_{12} \bar{q}_{12}^{\prime}=0$.

From (40) and (41) we find

so that either

$$
\bar{\Delta}_{1}-\bar{\Delta}_{2}=\bar{p}_{12} \bar{q}_{12}\left(\bar{p}_{11}-\bar{p}_{22}\right)=0,
$$

$$
\bar{p}_{12}=0, \quad \bar{q}_{12}=0, \quad \text { or } \quad \bar{p}_{11}-\bar{p}_{22}=0 .
$$

We may then replace conditions (40) and (41) by one of the pairs of equations

$$
\begin{gathered}
\bar{\Delta}_{1}=0, \quad \bar{p}_{12}=0, \\
\bar{\Delta}_{1}=0, \quad \bar{q}_{12}=0, \\
\bar{\Delta}_{1}=0, \quad \bar{p}_{11}-\bar{p}_{22}=0 .
\end{gathered}
$$

(a) If $\bar{p}_{12}=0, \bar{\Delta}_{1}=0$ reduces to $q_{12}=0$. Now from

we have as before

$$
\bar{p}_{12}=\frac{1}{2}\left(\frac{p_{12}^{\prime}}{p_{12}}-\frac{p_{21}^{\prime}}{p_{21}}\right)=0
$$

$$
\frac{p_{12}^{\prime \prime}}{p_{12}}-\frac{3}{2}\left(\frac{p_{12}^{\prime}}{p_{12}}\right)^{2}=2\left(q_{22}-\frac{\Delta_{1}}{p_{12}^{2}}\right)=\frac{p_{21}^{\prime \prime}}{p_{21}}-\frac{3}{2}\left(\frac{p_{21}^{\prime}}{p_{21}}\right)^{2}=2\left(q_{11}-\frac{\Delta_{2}}{p_{21}^{2}}\right),
$$

or

$$
\frac{\Delta_{2}}{p_{21}^{2}}-\frac{\Delta_{1}}{p_{12}^{2}}=q_{11}-q_{22}
$$

But this result substituted in $q_{12}=0$ gives $q_{11}-q_{22}=0$, that is,

$$
\theta_{4}=\left(u_{11}-u_{22}\right)^{2}=0,
$$


and this together with $u_{12}=u_{21}=0$ reduces $S$ to a quadric,* a surface for which both flecnode curve and complex curve are indeterminate.

(b) If $\bar{q}_{12}=0, \bar{\Delta}_{1}=0$ reduces to either $\bar{p}_{12}=0$ or $\bar{q}_{11}=0$. The first possibility has already been treated in $(a)$. From $\bar{q}_{11}=0$ and $\bar{q}_{12}=0$ we conclude

$$
\begin{aligned}
& \bar{q}_{11}+\bar{q}_{12}=q_{11}-\frac{1}{2} \frac{p_{21}^{\prime \prime}}{p_{21}}+\frac{3}{4}\left(\frac{p_{21}^{\prime}}{p_{21}}\right)^{2}=0, \\
& \bar{q}_{11}-\bar{q}_{12}=q_{22}-\frac{1}{2} \frac{p_{12}^{\prime \prime}}{p_{12}}+\frac{3}{4}\left(\frac{p_{12}^{\prime}}{p_{12}}\right)^{2}=0,
\end{aligned}
$$

and these equations are equivalent to (10). Whence the two branches of the flecnode curve are distinct, non-rectilinear, plane curves.

(c) $\bar{p}_{11}-\bar{p}_{22}=0$ reduces to $\sqrt{p_{12} p_{21}}=0$, that is, either $p_{12}=0$ or $p_{21}=0$, a situation already considered.

We conclude then that the converse of Theorem I holds.

The question of the extent to which a ruled surface is determined by the condition that the two branches of its flecnode curve shall be distinct, nonrectilinear, plane curves may be answered by the following construction.

Let $C$ be any given plane curve. On $C$ let any set of points $P_{1}, \cdots, P_{5}, \ldots$ be taken, corresponding for instance to values of the parameter $\xi$ differing by $\Delta \xi$. Through each of the points $P_{1}, P_{2}, P_{3}$, draw an arbitrary line and let $Q_{1}, Q_{2}, Q_{3}$, be the points of intersection of these three lines $g_{1}, g_{2}, g_{3}$, with an arbitrary plane $\chi$. Through $P_{1}$ and $Q_{1}$ respectively draw the lines $f_{1}^{\prime}$ and $f_{1}^{\prime \prime}$ which intersect $g_{1}, g_{2}, g_{3}$. The plane determined by $f_{1}^{\prime}$ and $P_{4}$ will intersect $f_{1}^{\prime \prime}$. This point of intersection, together with $P_{4}$ will determine a line $g_{4}$ which intersects both $f_{1}^{\prime}$ and $f_{1}^{\prime \prime}$ and cuts the plane $\chi$ in a point $Q_{4}$. Through $P_{2}$ and $Q_{2}$ respectively draw the lines $f_{2}^{\prime}$ and $f_{2}^{\prime \prime}$ which intersect $g_{2}, g_{3}, g_{4}$. The plane determined by $f_{2}^{\prime}$ and $P_{5}$ will intersect $f_{2}^{\prime \prime}$. This point of intersection, together with $P_{5}$ will determine a line $g_{5}$ which intersects both $f_{2}^{\prime}$ and $f_{2}^{\prime \prime}$ and cuts the plane $\chi$ in a point $Q_{5}$. Proceed thus, locating each time a new point in the plane $\chi$. The sets of lines $f_{i}^{\prime}$ and $f_{i}^{\prime \prime}$ are such that each line of either set intersects four successive lines of the set $g_{i}$. When $\Delta \xi$ approaches zero the set of lines $g_{i}$ will constitute a ruled surface $S$, while the sets of lines $f_{i}^{\prime}$ and $f_{i}^{\prime \prime}$ will also constitute ruled surfaces $F^{\prime}$ and $F^{\prime \prime}$ such that each line of either surface will be a four-point tangent to $S$, those of $F^{\prime}$ having as locus of their points of tangency the curve $C$ and those of $F^{\prime \prime}$ having as locus of their points of tangency the locus of the points $Q_{i}$. $S$ will therefore be a ruled surface whose flecnode curve consists of two plane branches.

The arbitrary choice of the plane $\chi$ should, in the analysis, give rise to three constants of integration, while the three lines $g_{1}, g_{2}, g_{3}$, should give rise to

*W., p. 150. 
six additional constants, making nine in all. We may exhibit these nine constants as follows. We may think of the invariants $\bar{\theta}_{3}$ and $\bar{\Theta}_{8}$ of $C$ as given functions of a parameter $\xi$ since these two invariants are sufficient to determine a plane curve except for projective transformations, ${ }^{*}$ and since when a parametric representation of a plane curve $C$ is given, the invariants are known functions of the parameter. We wish to identify the curve $C$ with the branch $C_{y}$ of the flecnode curve of the surface $S$, where the invariants $\theta_{3}$ and $\theta_{8}$ of $C_{y}$ and the coefficients of system $(F)$ are thought of as unknown functions of $x$. We may assume that the independent variable $x$ of $(F)$ is so chosen as to make $q_{22}-q_{11}=1$ and we will then have for the determination of these unknowns, from $(8 a)$, the conditions

$$
p_{12}^{\prime}-2 q_{12}=0, \quad p_{21}^{\prime}-2 q_{21}=0,
$$

from $(S a)$ and $(10)$

$$
p_{12}^{2}\left(1+q_{11}\right)-p_{12} q_{12}^{\prime}+3 q_{12}^{2}=0, \quad p_{21}^{2} q_{11}-p_{21} q_{21}^{\prime}+3 q_{21}^{2}=0,
$$

$$
\frac{p_{12}^{\prime}}{p_{12}}+\theta_{3}=0
$$

The invariants $\theta_{3}$ and $\theta_{8}$ of $C_{y}$ are connected by the relation $\dagger$

$$
\theta_{8}=6 \Theta_{3} \theta_{3}^{\prime \prime}-7 \Theta_{3}^{\prime 2}-27 P_{2} \theta_{3}^{2},
$$

which, by (17), (17a), and (10) becomes

$$
6 \Theta_{3} \Theta_{3}^{\prime \prime}-7 \Theta_{3}^{\prime 2}+27\left[\frac{1}{3}\left(p_{12} p_{21}-4 q_{11}\right)-1\right] \Theta_{3}^{2}-\Theta_{8}=0 .
$$

To complete the identification of $C$ and $C_{y}$ we have the two relations

$$
\bar{\theta}_{8} \xi^{8}=\theta_{8}, \quad \bar{\theta}_{3} \xi^{3}=\theta_{3},
$$

which must hold when the independent variable is transformed from $\xi$ to $x$. By a suitable combination of equations (42) and (43), $q_{11}, q_{12}, q_{21}$ may be eliminated and a new equation obtained involving only $p_{12}, p_{21}$ and their first and second derivatives. $\theta_{3}$ may be eliminated between (44) and the second of (46) and an equation of the first order in $\xi$ and $p_{12}$ obtained. By means of the second of (43) and equations (46), $\Theta_{3}, \Theta_{8}$, and $q_{11}$ may be eliminated from (45) and an equation of the third order involving $\xi, p_{12}, p_{21}$ obtained. The solution of these three equations in the three unknowns $\xi, p_{12}, p_{21}$, is a problem of the sixth order involving six constants of integration. When $\xi, p_{12}, p_{21}$ have been found, all the remaining coefficients of system $(F)$ may be obtained without further integration. The existence of a six-parameter family of

*W., p. 61 .

† W., p. 59 . 
surfaces each of which has the given curve $C$ for one branch of its flecnode curve, is thus established. But to the surfaces of this family must be added all those which may be obtained from them by the most general projective transformation which leaves the points of the plane of $C$ invariant. This transformation involves three additional parameters, thus completing the count.

\section{Case of COINCident plane BRANChes of the flecnode CURVE}

When the two branches of the flecnode curve are coincident we may no longer make use of our system of differential equations in the form $(F)$. If we choose the curve with which both branches of the flecnode curve coincide for one directrix curve, $C_{y}$, and any asymptotic curve of $S$ for the other directrix curve, we shall have

$$
\Theta_{4}=0, \quad u_{12}=0, \quad p_{21}=0 .^{*}
$$

We may further assume that $p_{11}=p_{22}=0$ without disturbing conditions (47). From (47), (4), (5a), and (5d) we find

$$
\Theta_{4}=\left(u_{11}-u_{22}\right)^{2}=16\left(q_{22}-q_{11}\right)^{2}=0,
$$

so that $q_{11}=q_{22}$. System $(A)$ now becomes

$\left(F_{1}\right) \quad y^{\prime \prime}+p_{12} z^{\prime}+q_{22} y+\frac{1}{2} p_{12}^{\prime} z=0, \quad z^{\prime \prime}+q_{21} y+q_{22} z=0$.

Corresponding to equations (5) we have

(48a) $u_{11}=u_{22}=-4 q_{22}, \quad u_{12}=0, \quad u_{21}=-4 q_{21}$,

$$
\begin{gathered}
v_{11}=-8 q_{22}^{\prime}-4 p_{12} q_{21}, \quad v_{12}=0, \quad v_{21}=-8 q_{21}^{\prime}, \\
v_{22}=-8 q_{22}^{\prime}+4 p_{12} q_{21}, \\
u_{11}=-16 q_{22}^{\prime \prime}-8 p_{12}^{\prime} q_{21}-16 p_{12} q_{21}^{\prime}, \quad w_{12}=8 p_{12}^{2} q_{21}, \\
u_{21}=-16 q_{21}^{\prime \prime}, \quad w_{22}=-16 q_{22}^{\prime \prime}+8 p_{12}^{\prime} q_{21}+16 p_{12} q_{21}^{\prime}, \\
I=u_{11}+u_{22}=-8 q_{22}, \quad J=u_{11} u_{22}=16 q_{22}^{2}, \\
K=v_{11} v_{22}=64 q_{22}^{\prime 2}-16 p_{12}^{2} q_{21}^{2} .
\end{gathered}
$$

The invariants given by (4) may now be calculated. They are

$$
\begin{gathered}
\theta_{4}=0, \quad \theta_{4,1}=0, \quad \theta_{9}=2^{8} p_{12}^{3} q_{21}^{3}, \quad \theta_{10}=0, \\
\theta_{6}=-2^{4} 3^{2} p_{12}^{2} q_{21}^{2}, \dagger
\end{gathered}
$$

Let the independent variable now be so chosen+ that

\footnotetext{
*W., p. 142 .

†n general, $\Theta_{6}$ is defined by $\theta_{6}=2 I \Theta_{4}+5\left(K-I^{\prime 2}\right)+4\left(K+I I^{\prime \prime}-2 J^{\prime \prime}\right)$.

$\ddagger W$., p. 172.
}$$
u_{11}+u_{22}=-8 q_{22}=0 \text {, }
$$ 
and at the same time let us suppose that $C_{y}$ is a plane curve. The first of equations (10) becomes

from which we obtain

$$
\frac{1}{2} p_{12} p_{12}^{\prime \prime}-\frac{3}{4} p_{12}^{\prime 2}=0,
$$

$$
p_{12}=\frac{4}{\left(c x+c_{1}\right)^{2}} .
$$

The coefficients of $\left(F_{1}\right)$ now involve one arbitrary function $q_{21}$ and two constants of integration. But one of the latter is not essential, for the transformation

$$
\eta=y, \quad \zeta=c z, \quad \xi=x+c_{1} / c,
$$

leaves unchanged all the conditions thus far imposed and serves to remove $c_{1}$. System $\left(F_{1}\right)$ now takes the form

$$
\eta^{\prime \prime}+\frac{4}{c^{3} \xi^{2}} \zeta^{\prime}+\frac{4}{c^{3}} \frac{\xi^{3}}{} \zeta=0, \quad \zeta^{\prime \prime}+c q_{21} \eta=0,
$$

and for this system we find

$$
\Theta_{4}=0, \quad \Theta_{6}=-\frac{3^{2} 2^{8} q_{21}^{2}}{c^{4} \xi^{4}}, \quad \Theta_{9}=\frac{2^{14} q_{21}^{3}}{c^{6} \xi^{6}}, \quad \Theta_{4 \cdot 1}=0, \quad \Theta_{10}=0 .
$$

If $\theta_{6}$ and $\theta_{9}$ are regarded as given functions of $\xi$ then the coefficients of system $\left(F_{1}\right)$ are determined except for one constant of integration.

Of special interest in the case under consideration is the secondary developable of the flecnode curve.* Through each point of the flecnode curve there pass four lines of special interest, the generator $P_{y} P_{z}$ of $S$, the flecnode tangent $P_{y} P_{\rho}$, the tangent to the flecnode curve $P_{y} P_{y^{\prime}}$, and the harmonic conjugate of the latter with respect to the first two. The locus of this last line is a developable surface called by Wilczynski the secondary developable of the flecnode curve. The tangents to the flecnode curve also generate a developable which is called the primary developable. Associated with these two developables is a one-parameter family of ruled surfaces $\dagger$ each of which has one branch of its flecnode curve in common with that of $S$. Moreover, the generators of these surfaces, at each point of their common flecnode curve, form an involution the double lines of which are the generators of the primary, and secondary, developables through that point.

The secondary developable is the locus of the line $P_{y} P_{\tau}^{\dagger}$ where

$$
\tau=y^{\prime}+p_{12} z,
$$

while the one-parameter family of ruled surfaces is generated by the line $P_{y} P_{0}$ where

*W., p. 233.

† W., p. 234.

$$
e=\tau+k y^{\prime}=(1+k) y^{\prime}+p_{12} z,
$$


any given surface of the family being obtained by holding $k$ fixed* and varying $x$. The system of differential equations for this family of ruled surfaces has for dependent variables $y$ and $e$ and for coefficients, functions of $k$, and of the coefficients of system $(F)$, or of system $\left(F_{1}\right)$, according as the two branches of the flecnode curve are distinct or coincident. For the latter case, if in addition to condition (47) we impose the condition that $C_{y}$ shall be plane, given by the first of equations (10), the coefficients in question are

$$
\begin{aligned}
P_{11} & =-\frac{1+k}{2 k} \vartheta, \quad P_{12}=-\frac{1}{k}, \\
Q_{11} & =-\frac{1}{2 k}\left(\vartheta^{\prime}-\frac{1}{2} \vartheta^{2}\right), \quad Q_{12}=\frac{\vartheta}{2 k}, \\
(50 a) \quad P_{21} & =\frac{(1+k)(1-3 k)}{4 k} \vartheta^{2}+(1+k) \vartheta^{\prime}, \quad P_{22}=\frac{1-3 k}{2 k} \vartheta, \\
Q_{21} & =-k p_{12} q_{21}+\frac{1}{2}(1+k)\left(\vartheta^{\prime \prime}-\vartheta \vartheta^{\prime}\right) \\
Q_{22} & =\frac{2 k-1}{4 k} \vartheta^{2}-\frac{1}{2} \vartheta^{\prime},
\end{aligned}
$$

where $\vartheta=p_{12}^{\prime} / p_{12} \cdot \dagger$

The invariants of this family of surfaces may now be obtained by applying (4) and (5). They do not involve $k$ and are precisely those of the surface $S$ given by (49). Since $\theta_{4}=0$, the flecnode curve on each surface of this family has coincident plane branches. But there is a further important property of this family. If we consider any two surfaces $S_{k_{1}}$ and $S_{k_{2}}$, then the system of equations whose integrating ruled surface is $S_{k_{1}}$, is transformed, coefficient for coefficient, by

$$
y=k_{2} \bar{y}, \quad e=\frac{1}{2}\left(k_{1}-k_{2}\right) \vartheta \bar{y}+k_{1} \bar{e},
$$

into the system whose integrating ruled surface is $S_{k_{2}}$, that is, the surfaces of the family are projectively related. We have then

Theorem II: If the two branches of the flecnode curve of a ruled surface $S$ coincide in a plane curve, then there exists a one-parameter family of mutually projective ruled surfaces each having the two branches of its flecnode curve coinciding with that of $S$, and to this family $S$ and its flecnode surface belong.

The curve $C_{y}$ has been assumed to be plane and therefore the primary de-

${ }^{*}$ It is of interest to note that the cross ratio of the lines $P_{y} P_{\tau}, P_{y} P_{e}, P_{y} P_{y \prime}, P_{y} P_{z}$, is $-k$.

$\dagger S$ and its flecnode surface $F$ are obtained by taking $k$ equal respectively to -1 and 1 . 
velopable reduces to the plane of $C_{y}$. From (50), by differentiation and the use of the first of $\left(F_{1}\right)$, we find

$$
q_{22} y+\frac{1}{2} \frac{p_{12}^{\prime}}{p_{12}} y^{\prime}-\frac{1}{2} \frac{p_{12}^{\prime}}{p_{12}} \tau+\tau^{\prime}=0,
$$

so that $y, y^{\prime}, \tau, \tau^{\prime}$, satisfy a linear relation of the form

where

$$
\lambda y+\mu y^{\prime}+\nu \tau+\rho \tau^{\prime},
$$

$$
\lambda=q_{22}, \quad \mu=\frac{1}{2} \frac{p_{12}^{\prime}}{p_{12}}, \quad \nu=-\frac{1}{2} \frac{p_{12}^{\prime}}{p_{12}}, \quad \rho=1 .
$$

We further observe that

$$
\mu^{\prime} \rho-\mu \rho^{\prime}-\lambda \rho+\mu \nu=\frac{1}{2} \frac{p_{12}^{\prime \prime}}{p_{12}}-\frac{3}{4}\left(\frac{p_{12}^{\prime}}{p_{12}}\right)^{2}-q_{22}=0,
$$

in view of the first of equations (10). But (51) is the condition that the developable generated by $P_{y} P_{\tau}$ shall degenerate into a cone whose vertex is given by

$$
\bar{g}=\mu y+\rho \tau=\frac{1}{2} \frac{p_{12}^{\prime}}{p_{12}} y+y^{\prime}+p_{12} z=\frac{1}{2 p_{12}}\left(p_{12} \rho+p_{12}^{\prime} y+p_{12}^{2} z\right) .^{*}
$$

We have shown that when the two branches of the flecnode curve coincide in a non-rectilinear, plane curve, the secondary developable of this curve becomes a cone. Let us assume only that one of the two branches, $C_{y}$ say, of the flecnode curve is plane and that its secondary developable is a cone. The condition for this is $\dagger$

$$
\lambda=2 p_{12}^{2}\left(q_{22}-q_{11}\right)=0 .
$$

But since $p_{12} \neq 0$, it follows that $q_{22}-q_{11}=0$ and hence that $\theta_{4}=0$. The two branches of the flecnode curve therefore coincide. We may now combine the last two results in

THEOREM III: In order that the two plane branches of the flecnode curve of a ruled surface may coincide it is necessary and sufficient that the secondary developable of one of these curves be a cone.

Let us think for a moment of the flecnode curve as being composed of two distinct branches not necessarily plane. The edges of regression of the secondary developables of these two branches are the loci of the points whose expressions are

$$
g=p_{12}^{\prime} y+p_{12}^{2} z+p_{12} \rho, \quad h=p_{21}^{2} y+p_{21}^{\prime} z+p_{21} \sigma .+
$$

\footnotetext{
*W., p. 131.

$\dagger$ W., p. 236.

$\ddagger$ W., p. 235.
} 
Now the equation of the quadric osculating $S$ along $L_{y z}$ is

$$
x_{1} x_{4}-x_{2} x_{3}=0 \text {.* }^{*}
$$

The equation of the polar plane of the point $P_{g}$ with respect to this quadric is

$$
p_{12} x_{2}+p_{12}^{2} x_{3}-p_{12}^{\prime} x_{4}=0,
$$

and that of the polar plane of the point $P_{h}$ is

$$
p_{21} x_{1}-p_{21}^{\prime} x_{3}+p_{21}^{2} x_{4}=0 \text {. }
$$

But these are exactly the planes which osculate $C_{y}$ and $C_{z}$ at $P_{y}$ and $P_{z}$ respectively, as given by (38) and (39). In other words, the edges of regression of the secondary developables of the two branches of the flecnode curve of $S$ are the loci of the poles of the planes osculating the flecnode curve, taken with respect to the quadrics which osculate $S$. This theorem has been stated without proof by Tzitzeica. $\dagger$

If now $C_{y}$ and $C_{z}$ coincide in a plane curve, the osculating planes coincide with the plane of this curve and the secondary developable becomes a cone the vertex of which is the common pole of the plane of this curve taken with respect to the osculating quadrics of $S$. The expression for this point given by (50) is of course a multiple of that given by the first of (53). $\neq$ We make use of this pole and polar relation in a construction of the surface.

Let $C$ be any given plane curve. On $C$ let points $P_{1}, \cdots, P_{5}, \cdots$ be taken corresponding to values of the independent variable differing by $\Delta \xi$. Through $P_{1}$ and any point $P$ not in the plane of $C$, pass the line $P P_{1}$. This line, together with the tangent to $C$ at $P_{1}$, determines a plane $P P_{1} T_{1}$. In this plane draw any line $g_{1}$ through $P_{1}$. In the same way through $P_{2}$ and $P_{3}$ draw lines $g_{2}$ and $g_{3}$ lying respectively in the planes $P P_{2} T_{2}$ and $P P_{3} T_{3}$. Through $P_{1}$ draw the line $f_{1}$ which cuts $g_{2}$ and $g_{3}$. It will intersect the plane $P_{4} P_{4}$ in a point which with $P_{4}$ will determine a line $g_{4}$. Through $P_{2}$ draw the line $f_{2}$ which cuts $g_{3}$ and $g_{4}$. It will cut the plane $P P_{5} T_{5}$ in a point which

* W., p. 191.

$\dagger$ Tzitzeica, C o m p t e s R en d u s, July, 1908, p. 173.

$\ddagger$ In the one-parameter family of ruled surfaces whose differential equations have the coefficients given by $(50 a)$, the surfaces $S_{k}$ and $S_{-k}$ bear the same relation to the primary and secondary developables of $C_{y}$ as do $S$ and $F$. It is therefore to be expected that $P_{z}$ would be the pole of the plane of $C_{y}$ with respect to each of the quadrics $H_{k}$ osculating $S_{k}$. This is easily verified, for the equation of $H_{k}$ is found to be

$$
x_{1} x_{4}-\frac{1+k^{2}}{2} x_{2} x_{3}=\frac{1}{2}\left(k^{2}-1\right)\left(\begin{array}{c}
x_{2}^{2} \\
2 p_{12}
\end{array}+\frac{p_{12} x_{3}^{2}}{2}+\frac{p_{12}^{\prime 2} x_{4}^{2}}{2 p_{12}^{3}}-\frac{p_{12}^{\prime}}{p_{12}^{2}} x_{2} x_{4}-\frac{p_{12}^{\prime}}{p_{12}} x_{3} x_{4}\right),
$$

and the polar plane of $P_{g}$ with respect to $H_{k}$ is given by the equation

$$
p_{12} x_{2}+p_{2}^{2} x_{3}-p_{12}^{\prime} x_{4}=0 .
$$

$P_{g}$ is therefore the pole of the plane of $C_{y}$ with respect to an $\infty^{2}$ of osculating quadrics. 
with $P_{5}$ will determine a line $g_{5}$. So proceed, obtaining alternately new lines $f$ and $g$. The two sets of lines $g$ and $f$ are such that each line of either set intersects four consecutive lines of the other set. Now let $\Delta \xi$ approach zero. As the points $P_{i-1}, P_{i}, P_{i+1}$ approach coincidence, the quadric determined by the three lines $g_{i-1}, g_{i}, g_{i+1}$, approaches a limit quadric $H_{i}$. Moreover, with respect to this quadric $P$ and the plane of $C$ have the pole and polar relation since $P$ is the point common to the planes tangent to $H_{i}$ at $P_{i-1}, P_{i}, P_{i+1}$. At the same time the two sets of lines $g_{i}$ and $f_{i}$ will approach two ruled surfaces $S$ and $F$ each of which is one sheet of the flecnode surface of the other, and $C$ will be one branch of the flecnode curve for each surface. The quadrics $H_{i}$ will osculate $S$ along the generators $g_{i}$ and the plane of $C$ will have with respect to all these osculating quadrics the common pole $P$. Since the locus of the poles of the plane of $C$ with respect to the $H_{i}$ is the edge of regression of the secondary developable of $C$, this developable is a cone and hence $S$, and therefore $F$, have the two branches of their common flecnode curve coincident and plane.

The way in which $g_{1}, g_{2}, g_{3}$ have been taken should in the analysis give rise to three constants while the choice of an arbitrary point $P$ should produce three more, making six in all. Under the conditions

$$
\theta_{4}=p_{11}=p_{21}=p_{22}=u_{12}=0,
$$

and with $C_{y}$ a plane curve, we have from (10) and $\left(F_{1}\right)$

$$
p_{12}^{2} q_{22}-p_{12} q_{12}^{\prime}+3 q_{12}^{2}=0, \quad q_{11}-q_{22}=0, \quad p_{12}^{\prime}-2 q_{12}=0 .
$$

By an appropriate choice of the independent variable of the surface, we may make $\theta_{3}=1{ }^{*}$ When this is done it is easily shown that the invariants $\theta_{3}$ and $\theta_{8}$ of $C_{y}$ satisfy the relations

$$
\begin{gathered}
p_{12} q_{21}=-\Theta_{3}=-1, \\
\Theta_{8}+36 q_{22} \Theta_{3}^{2}+7 \Theta_{3}^{\prime 2}-6 \Theta_{3} \Theta_{3}^{\prime \prime}=\Theta_{8}+36 q_{22}=0 .
\end{gathered}
$$

In order to identify the curves $C$ and $C_{y}$ relations (46) must be satisfied. By making use of the first and third of (54), the second of (55) and the first of (46), we may eliminate $q_{12}, q_{22}$, and $\Theta_{8}$ and obtain an equation of the second order involving $p_{12}$ and $\xi$. The second of (46), in view of the choice of the independent variable, reduces to an equation of the first order in $\xi$ alone. The solution of these two equations in the unknowns $p_{12}, \xi$, involves three constants of integration. When $p_{12}$ and $\xi$ have been found the remaining unknowns may be obtained without further integration.

\footnotetext{
* We may always do this unless $\theta_{3} \equiv 0$. But if $\theta_{3} \equiv 0$ either $p_{12}=0$ or $q_{21}=0$ from (55). Since we exclude here the case where $C_{y}$ is a straight line, $p_{12} \neq 0$, so that we must have $q_{21}=0$. But this makes $u_{21}=0$ by (48a) and hence $S$ is a quadric.
} 
The existence of a three-parameter family of ruled surfaces each of which has the two branches of its flecnode curve coinciding with $C$, is thus established. But to the surfaces of this family must be added all those which may be obtained from them by the three-parameter projective transformation which leaves the points of the plane of $C$ invariant. The six constants of the construction are thus accounted for.

We may think of each surface of the three-parameter family first obtained as being determined by a pair of directrix curves consisting of the curve $C$ and one of the asymptotic curves of the surface. Since on each surface there is a single infinity of asymptotic curves, the $\infty^{3}$ of surfaces gives rise to a fourfold infinity of asymptotic curves. If the constants involved in calculating the coefficients of system $\left(F_{1}\right)$ are thought of as determining the directrix curves of the surface rather than the surface itself, then it would seem that four such constants are required. But it has been shown that there exists a single infinity of mutually projective surfaces having $C$ for common flecnode curve and all having the same cone for secondary developable. The double infinity of asymptotic curves belonging to this family of surfaces are themselves mutually projective and hence may be accounted for by a single constant in the calculation of the coefficients of system $\left(F_{1}\right)$.

\section{RECTILINEAR BRANCHES OF THE FLECNODE CURVE.}

The case where one, or both, branches of the flecnode curve are straight lines has been considered in detail by Wilczynski.* It is worth while however to call attention to one or two matters of interest.

Let $p_{21}=0$. Then system $(F)$ becomes

$$
y^{\prime \prime}+p_{12} z^{\prime}+q_{11} y+\frac{1}{2} p_{12} z^{\prime}=0, \quad z^{\prime \prime}+q_{22} z=0,
$$

so that $C_{z}$ is a straight line. Moreover, by (7), $\theta_{9}=0$ and $\theta_{10}=0$. If we think of the non-vanishing invariants $\theta_{4}$ and $\theta_{4 \cdot 1}$ as given functions of $x$, it is possible to determine $q_{11}$ and $q_{22}$ from the first two of equations (7). But $p_{12}$ remains as an arbitrary function, that is, the invariants do not suffice to characterize the ruled surfaces. $\dagger$ If however we suppose $C_{y}$ to be a plane curve, then from the first of equations (10), $p_{12}$ may be determined except for two constants of integration.

We have seen that a ruled surface cannot have two non-degenerate conics for the two branches of its flecnode curve. But these two branches may consist of a straight line and a conic as we shall see.

Under the conditions $p_{11}=p_{21}=p_{22}=u_{12}=u_{21}=0$, the value of the

*W., ch. VIII.

$\dagger$ W., p. 171. 
invariant of weight three of $C_{y}$ is given by (18). For $\theta_{3}=0$ we get on integrating

$$
p_{12}=c\left(q_{22}-q_{11}\right),
$$

or, if we suppose the independent variable to be so chosen that

$$
u_{11}-u_{22}=4\left(q_{22}-q_{11}\right)=1,
$$

then $p_{12}=\frac{1}{4} c . \quad$ The first of equations (10) then gives $q_{22}=0$ so that $q_{11}=-\frac{1}{4}$ and (56) becomes

$$
y^{\prime \prime}+\frac{1}{4} c z^{\prime}-\frac{1}{4} y=0, \quad z^{\prime \prime}=0 .
$$

Equations (57) are indeed a special case of equations (28).

We proceed to obtain the cartesian equation of a ruled surface which has a straight line and a conic for the two branches of its flecnode curve. For convenience let $c=1$. Then a fundamental system of solutions of (57) is

$$
\begin{array}{llll}
y_{1}=e^{1 x}, & y_{2}=e^{-\frac{1}{2} x}+1, & y_{3}=e^{\frac{1}{3} x}-1, & y_{4}=e^{-\frac{1}{2} x}, \\
z_{1}=1, & z_{2}=x, & z_{3}=-x, & z_{4}=1 .
\end{array}
$$

An arbitrary point on the line $L_{y z}$ is given by

$$
\begin{aligned}
x_{1} & =\lambda e^{\frac{1}{2} x}+\mu, & x_{2} & =\lambda\left(e^{-\frac{1}{2} x}+1\right)+\mu x, \\
x_{3} & =\lambda\left(e^{\frac{1}{2} x}-1\right)-\mu x, & x_{4} & =\lambda e^{-\frac{1}{2} x}+\mu .
\end{aligned}
$$

We have

$$
\begin{aligned}
& X_{1}=x_{1}+x_{2}+x_{3}-x_{4}=2 \lambda e^{\frac{3}{2} x}, \quad X_{3}=x_{1}-x_{2}-x_{3}+x_{4}=2 \mu, \\
& X_{2}=-x_{1}+x_{2}+x_{3}+x_{4}=2 \lambda e^{-\frac{1}{2} x}, \quad X_{4}=x_{1}+x_{2}-x_{3}-x_{4} \\
& =2(\lambda+\mu x), \\
& \frac{X_{1}}{\bar{Y}_{3}}=\frac{\lambda}{\mu} e^{\frac{1}{2} x}, \quad \frac{X_{2}}{X_{3}}=\frac{\lambda}{\mu} e^{-1 x}, \quad \frac{X_{4}}{X_{3}}=\frac{\lambda}{\mu}+x, \quad \frac{X_{1} X_{2}}{X_{3}^{2}}=\frac{\lambda^{2}}{\mu^{2}}, \quad \frac{X_{1}}{X_{2}}=e^{x},
\end{aligned}
$$

whence

which becomes

$$
\frac{X_{4}}{X_{3}}= \pm \frac{\sqrt{X_{1} X_{2}}}{X_{3}}+\log \frac{X_{1}}{X_{2}}
$$

$$
\xi=(\zeta-\eta \log \xi)^{2}
$$

where $X_{2} / X_{1}=\xi, X_{3} / X_{1}=\eta$ and $X_{4} / X_{1}=\zeta$ are interpreted as the cartesian coördinates of a point in three space. The introduction of $X_{i}$ in place of $x_{i}$ amounts merely to a projective transformation and this does not change the character of the curves involved. It is easy to show that for the above surface the flecnode curve is made up of the parabola $\zeta^{2}=\xi$ in which it is cut 
by the $\xi \zeta$-plane, and the line at infinity common to the set of planes perpendicular to the $\xi$-axis. The rulings of the surface are the straight lines

$$
\begin{aligned}
& \xi=k, \quad \zeta=\eta \log k \pm \sqrt{k} . \\
& \text { DUalization OF THE PROBLEM. }
\end{aligned}
$$

The adjoint system of system $(F)$, whose integrating ruled surface is the space dual of $S, *$ has the coefficients

(59) $\quad P_{i k}=p_{i k}, \quad Q_{12}=q_{12}, \quad Q_{21}=q_{21}, \quad Q_{11}=q_{22}, \quad Q_{22}=q_{11}$.

If $\Sigma$ is the space dual of $S$, then to each point, line, plane, of $S$ there corresponds a plane, line, point, of $\Sigma$.

Corresponding to the flecnode tangents of $S$, thought of as four point tangents, there will be a set of tangents to $\Sigma$, each one lying in four successive tangent planes, that is, having four consecutive points (the points of tangency of the four planes) in common with $\Sigma$. Hence the flecnode curve of $\Sigma$ corresponds to the flecnode curve of $S$.

The condition that all the points of the flecnode curve of $S$ shall lie in two distinct planes corresponds to the condition that all the planes tangent to $\Sigma$ along its flecnode curve shall form two distinct cones. Equations (10) may evidently be interpreted in either way, or, in view of (59), we may say that the conditions

$$
p_{12}^{2} q_{11}-\frac{1}{2} p_{12} p_{12}^{\prime \prime}+\frac{3}{4} p_{12}^{\prime 2}=0, \quad p_{21}^{2} q_{22}-\frac{1}{2} p_{21} p_{21}^{\prime \prime}+\frac{3}{4} p_{21}^{\prime 2}=0,
$$

are necessary and sufficient to insure that the tangent planes to $S$ along its flecnode curve shall envelope two distinct cones.

We may now dualize some of our preceding results.

There are no ruled surfaces the tangent planes to which along the flecnode curve, envelope two quadric cones.

There exists a single infinity of classes of mutually projective ruled surfaces having the property that their tangent planes drawn at points of their flecnode curves envelope two distinct cones, one of which is a quadric cone.

If the tangent planes to a ruled surface along its flecnode curve envelope two distinct cones, then the tangent planes at the points of its complex curve have this same property, and the vertices of these four cones form a harmonic range on which the vertices of the first two are separated by the vertices of the second two.

Since the invariants of the adjoint system are the same as those for system $(F)$ except that $\bar{\theta}_{9}=-\theta_{9} \dagger$ it results that when the branches of the flecnode

*W., p. 134.

†W., p. 143. 
curve of $S$ coincide, those of $\Sigma$ also are coincident. Moreover if the former constitute a plane curve, the tangent planes to $\Sigma$ along its flecnode curve, envelope a cone. But we have seen that this last is both necessary and sufficient to make the flecnode curve of $\Sigma$ a plane curve, for the envelope of the tangent planes along a branch of the flecnode curve is the secondary developable. We conclude therefore that if the two branches of the flecnode curve of a ruled surface coincide in a plane curve, the same is true of the dual surface. Moreover both surfaces have the dual property.

The University of Chicago, March, 1915.

\section{ERRATA, VOLUME 15}

Page 87. Burton H. Camp. Lebesgue integrals containing a parameter, with applications.

Page 106, line 2 from bottom, change last letter $\tau$ in line to $t_{1}$; last line, insert at end of sentence " and (a)." 